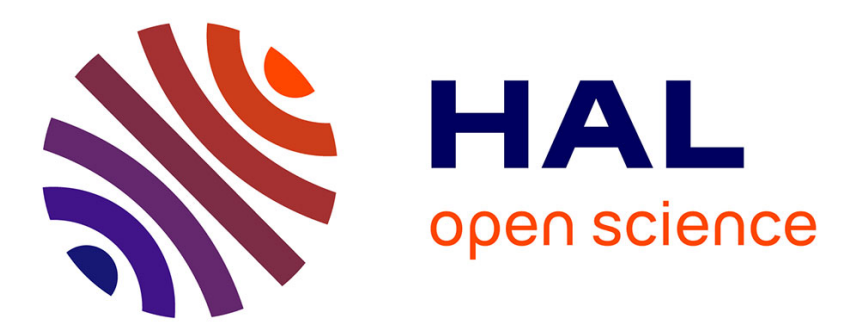

\title{
Biasing Smarter, Not Harder, by Partitioning Collective Variables into Families in Parallel Bias Metadynamics
}

\author{
Arushi Prakash, Christopher Fu, Massimiliano Bonomi, Jim Pfaendtner
}

\section{To cite this version:}

Arushi Prakash, Christopher Fu, Massimiliano Bonomi, Jim Pfaendtner. Biasing Smarter, Not Harder, by Partitioning Collective Variables into Families in Parallel Bias Metadynamics. Journal of Chemical Theory and Computation, 2018, 14 (10), pp.4985-4990. 10.1021/acs.jctc.8b00448 . hal-03136535

\section{HAL Id: hal-03136535 \\ https://hal.science/hal-03136535}

Submitted on 9 Feb 2021

HAL is a multi-disciplinary open access archive for the deposit and dissemination of scientific research documents, whether they are published or not. The documents may come from teaching and research institutions in France or abroad, or from public or private research centers.
L'archive ouverte pluridisciplinaire HAL, est destinée au dépôt et à la diffusion de documents scientifiques de niveau recherche, publiés ou non, émanant des établissements d'enseignement et de recherche français ou étrangers, des laboratoires publics ou privés. 


\title{
Biasing Smarter, Not Harder, By Partitioning
}

\section{Collective Variables Into Families in Parallel Bias}

\section{Metadynamics}

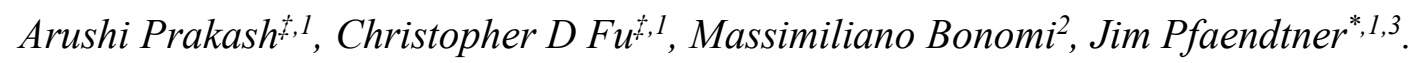

*Corresponding author (jpfaendt@uw.edu),.Equal contributions by authors

${ }^{1}$ Department of Chemical Engineering, University of Washington, Seattle, Washington 98195,

United States

${ }^{2}$ Department of Chemistry, University of Cambridge, Lensfield Road, Cambridge CB2 1EW, United Kingdom

${ }^{3}$ Senior Scientist, Pacific Northwest National Laboratory, Richland, Washington, United States

\begin{abstract}
Molecular simulations of systems with multiple copies of identical atoms or molecules may require the biasing of numerous, degenerate collective variables (CVs) to accelerate sampling. Recently, a variation of metadynamics (MetaD) named parallel bias metadynamics (PBMetaD) has been shown to make biasing of many CVs more tractable. We extended the PBMetaD scheme so that it partitions degenerate CVs into families that share the same bias potential, consequently expediting convergence of the free-energy landscape. We tested our method, named Parallel Bias MetaD with Partitioned Families, on 3, 21, and 78 CV systems and obtained an approximately proportional increase in convergence speed compared to standard PBMetaD.
\end{abstract}




\section{INTRODUCTION}

Molecular simulations have immense potential to provide crucial molecular- and nanoscale details of physical, chemical, and biological processes. However, simulations of slow molecular transitions, like protein folding/unfolding and chemical reactions, continue to be stymied due to high free-energy barriers and rugged free-energy landscapes that limit sampling. Several enhanced sampling methods, like metadynamics (MetaD), ${ }^{1,2}$ umbrella sampling, ${ }^{3}$ hyperdynamics, ${ }^{4}$ variationally enhanced sampling, ${ }^{5}$ and adaptive force biasing, ${ }^{6}$ have tried to alleviate this problem by applying a bias along predefined coarse-grained descriptors or collective variables (CVs) of the system to accelerate sampling. Since applying bias in a highdimensional $\mathrm{CV}$ space is often inefficient, researchers have traditionally resorted to choosing a minimal set of CVs. ${ }^{7}$

Identifying a small set of $\mathrm{CVs}$ that can effectively differentiate between relevant states of a system is a primary challenge for many enhanced sampling approaches. While a variety of CVselection methods, like time-lagged independent component analysis (TICA), ${ }^{8}$ reconnaissance metadynamics, ${ }^{9}$ and spectral gap optimization of order parameters (SGOOP), ${ }^{10}$ have recently been developed to address this challenge, researchers primarily still rely on their physicochemical intuition of the system to select optimal CVs. Frequently, more than one candidate CV is biased which presents the challenge of efficiently biasing them with limited computational resources. To this end, replica-based methods like multiple walker metadynamics, ${ }^{11}$ altruistic metadynamics, ${ }^{12}$ and the flying Gaussian method $^{13}$ have been developed to exploit parallel simulations that share the bias potential to accelerate sampling even further. However, these methods do not address the problem of the high dimensionality of the bias potential, which 
requires extensive sampling, due to the larger phase space that needs to be explored, for convergence.

Some MetaD-based methods have been formulated to address the challenge of biasing a large number of CVs. In bias exchange ${ }^{14}$ MetaD, a replica exchange approach is used, in which multiple replicas of the system, each biasing only one or few CVs, are simulated in parallel. Conformations of the system are periodically exchanged using a metropolis criterion. Other methods use several low dimensional bias potentials to bias individual CVs in lieu of a single high-dimensional bias potential. ${ }^{7,15}$ Parallel bias Metadynamics (PBMetaD) is one such method that has been used to bias 4-40 CVs in the same simulation. ${ }^{15-19}$

This letter introduces PBMetaD with partitioned families (PBMetaDPF) for systems that require biasing multiple CVs that share identical properties. As an example, a simulation can contain multiple copies of a protein in a box. Since these proteins are identical subunits, they are expected to share identical properties. Thus, a CV describing property $\mathrm{X}$ of the $\mathrm{n}^{\text {th }}$ subunit would also describe property $\mathrm{X}$ of any other subunit of the system. Consequently, a system with $\mathrm{N}$ identical subunits would contain N CVs describing property X for each subunit. These CVs can be considered as indistinguishable or degenerate, as their equilibrium probability distributions are identical. In PBMetaDPF, the gain in efficiency over PBMetaD is achieved by grouping degenerate $\mathrm{CVs}$ into one family so that $\mathrm{CVs}$ in the same family share and contribute to the same bias potential. Finally, the free-energy profile for each $\mathrm{CV}$ family can be determined using the standard MetaD integration and standard reweighting techniques ${ }^{20,21}$ can be used to study other degrees of freedom. The concept of a shared bias potential is inspired by multiple walker $\mathrm{MetaD}^{11}$ where the potential of a CV evolves by contributions from walkers of the CV in parallel 
replicas of the system. In contrast, in our method all contributions to this shared potential come from multiple CVs within one replica only.

\section{THEORY}

To highlight the differences between the PBMetaD and PBMetaDPF approaches, we will briefly review the theory of PBMetaD. In classical MetaD, or its well-tempered variant (WTMetaD), ${ }^{2}$ a single multidimensional bias potential is constructed as a function of userspecified CVs, where the dimensionality of the potential is equal to the number of CVs. In contrast, PBMetaD constructs multiple monodimensional bias potentials applied along each individual CV. The method uses a new scheme to permit the instantaneous application of an arbitrary number of bias potentials, which was shown to converge (empirically) to the exact underlying free-energy surfaces. ${ }^{15}$ To achieve this, the bias potential for the $i^{\text {th }} \mathrm{CV}\left(s_{i}\right)$, under the PBMetaD framework, is constructed through the following equation

$$
\begin{aligned}
& V_{G}\left(s_{i}, t\right)=\int_{0}^{t} d t^{\prime} W * \exp \left(-\frac{V_{G}\left(s_{i} R\left(t^{\prime}\right), t^{\prime}\right)}{k_{B} \Delta T}\right) * \exp \left(\frac{-\left(s_{i}(R(t))-s_{i}\left(R\left(t^{\prime}\right)\right)\right)^{2}}{2 \sigma^{2}}\right) * W_{P B}\left(s_{i}, t^{\prime}\right) \text { Eq. } 1 \\
& W_{P B}\left(s_{i}, t\right)=\frac{\exp \left(-\frac{V_{G}\left(s_{i} R(t), t\right)}{k_{B} T}\right)}{\sum_{j=1}^{n} \exp \left(-\frac{V_{G}\left(s_{j} R(t), t\right)}{k_{B} T}\right)} \quad \text { Eq. } 2
\end{aligned}
$$

where $W$ is the initial Gaussian height, $\sigma_{i}$ the width of the Gaussian, $d t^{\prime}$ the pace of Gaussian deposition, $k_{B}$ the Boltzmann constant, $T$ the system temperature, $\Delta T$ is an input parameter with units of temperature which controls the rate at which Gaussians are scaled down, and $n$ is the total number of CVs in the system. The first three terms in Eq. 1 follow the algorithm of a typical WTMetaD simulation where the Gaussian height is reduced as bias accumulates, while the last term, shown in Eq. 2, is a conditional weight to account for the effect of the bias deposited along 
the other CVs due the correlations among CVs. ${ }^{7}$ This conditional weight distributes a new Gaussian across all CVs, with the CVs with lower bias at the time of deposition receiving a larger contribution. In this algorithm, each bias potential evolves independently, and the only interaction occurs via the conditional weight term.

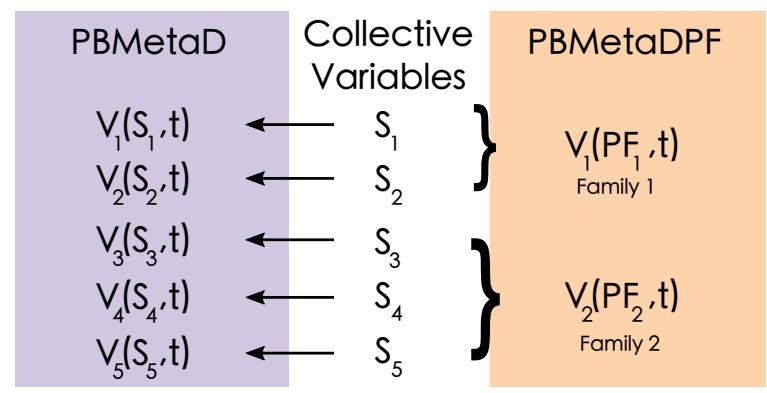

Scheme 1: Diagrammatic view of the differences between PBMetaD and PBMetaDPF sampling schemes. Under the PBMetaD biasing scheme, an individual bias potential is evolved for each $\mathrm{CV}$ and the $\mathrm{CV}$ only acts under its own potential. In contrast, the PBMetaDPF schemes allows for all of the members of a given family to contribute to the formation of a single bias potential that, in turn, acts on all of the members of a particular family.

While PBMetaD offers a more scalable way to perform high-dimensional sampling, converging numerous bias potentials independently can still require lengthy simulation times, as evidenced by Prakash et al. ${ }^{16}$ In PBMetaDPF, we expedite the convergence of these energy landscapes, which comprise indistinguishable particles, by partitioning degenerate CVs into families (PFs). For book-keeping purposes, we will refer to CVs as $s_{P F f-k}$, where $f$ refers to the $\mathrm{PF}$ it belongs to and $k$ refers to the $\mathrm{CV}$ index in that PF (e.g. $S_{P F 1-2}$ is the second $\mathrm{CV}$ belonging to $\mathrm{PF}$ one). CVs partitioned into the same PF deposit bias similar to the multiple walkers framework, ${ }^{11}$ where the Gaussians deposited along the different individual CVs of a particular PF all contribute to the formation of a single bias potential that acts on all the CVs belonging to 
that PF. The bias potential for any $\mathrm{CV}$ belonging to partitioned family PF1 $\left(s_{P F 1-x}\right.$; where $x$ is any member of family 1 ), which has $m$ members, is recovered through:

$V_{G}\left(s_{P F 1-x}, t\right)=\sum_{k=1}^{m} \int_{0}^{t} d t^{\prime} W * \exp \left(-\frac{V_{G}\left(s_{P F 1-k} R\left(t^{\prime}\right), t^{\prime}\right)}{k_{B} \Delta T}\right) * \exp \left(\frac{-\left(s_{P F 1-x}(R(t))-s_{P F 1-k}\left(R\left(t^{\prime}\right)\right)\right)^{2}}{2 \sigma^{2}}\right) * W_{P B}\left(s_{P F 1-k}, t^{\prime}\right)$ Eq. 3

Here, the bias potential of each family is constructed by the contributions of every member. In other words, the bias potential of the family acts on each member of the family. Consequently, only one free-energy profile is recovered per PF instead of recovering one free-energy profile for each $\mathrm{CV}$. Note that the denominator of the conditional weight term still sums over all the CVs biased in a system, as is done in regular PBMetaD.

\section{RESULTS}

\section{3-PARTICLE LENNARD-JONES SYSTEM}

To assess the accuracy and efficiency of our approach, we used a simple three-particle Lennard-Jones $(\mathrm{LJ})$ system $(\sigma=0.39 \mathrm{~nm}, \varepsilon=20 \mathrm{~kJ} / \mathrm{mol})$. For both PBMetaD and PBMetaDPF, we biased all three interatomic distances with initial Gaussian heights of $2.0 \mathrm{~kJ} / \mathrm{mol}$, Gaussian widths of $0.01 \mathrm{~nm}$, a bias factor of 10 , and a deposition pace of $1 \mathrm{ps}$. Sixteen independent biased simulations were run in the NVT ensemble for $2 \mu \mathrm{s}$. Further, to confirm that the PBMetaD framework is suitable for describing such systems, we performed parallel tempering (PT) simulations to provide an independent reference free-energy profile (see SI for details).

We monitored the root mean squared deviation (RMSD) of the free-energy profiles recovered from PBMetaD and PBMetaDPF relative to that obtained with PT to assess both convergence speed and accuracy. The RMSD between two profiles was defined as: ${ }^{2}$ 


$$
\operatorname{RMDS}\left(F_{r e f}, F\right)=\sqrt{\frac{1}{\Omega} \int d S\left[\left(F_{r e f}(S)-\bar{F}_{r e f}\right)-(F(S)-\bar{F})\right]^{2}} \text { Eq. } 4
$$

where $S$ is the $\mathrm{CV}$ value, $F_{\text {ref }}(S)$ and $F(S)$ are the two free-energy profiles being compared, $\bar{F}$ and $\bar{F}_{\text {ref }}$ are the free-energy averaged over the region $\Omega$. For the three-particle and 13-particle systems, the region of interest was defined as the $\mathrm{CV}$ space within $30 \mathrm{~kJ} / \mathrm{mol}$ of the global minimum of the reference PT profile, while for the seven-particle system the region of interest was defined as the $\mathrm{CV}$ space within $100 \mathrm{~kJ} / \mathrm{mol}$ of the global minimum of the reweighted interatomic distance profile from WTMetaD. For each PBMetaD simulation, we recovered three free-energy profiles, one for each interatomic distance. In the case of PBMetaDPF, a single freeenergy profile for a given simulation is naturally recovered because the method groups the three CVs into the same family.
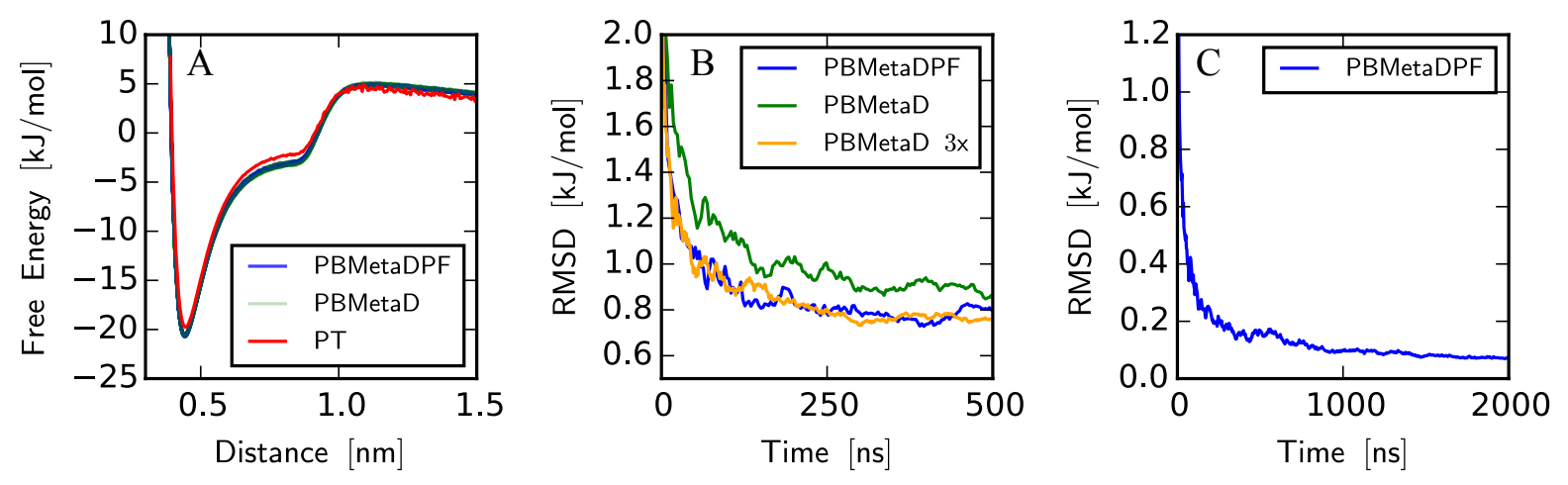

Figure 1: (A) Mean-aligned free-energy profiles of the interatomic distance between LJ particles. In total, the 16 PBMetaDPF profiles, the 48 PBMetaD profiles, and one parallel tempering (PT) profile are plotted. (B) The average RMSD of PBMetaDPF profiles (blue), PBMetaD profiles (green), and of PBMetaD with a projected convergence rate of three times faster (orange), all RMSD calculations are relative to the reference PT profile. (C) The average RMSD of PBMetaDPF relative to the converged PBMetaD profile over the course of the simulation. 
As shown in Figure 1A, both PBMetaD and PBMetaDPF accurately reproduced the freeenergy profile along the interatomic distance obtained with PT. The RMSD of the free-energy profile recovered from PBMetaDPF simulations is well-within $\mathrm{k}_{\mathrm{b}} \mathrm{T}(\sim 2.5 \mathrm{~kJ} / \mathrm{mol}$ at $300 \mathrm{~K})$ when compared to PT (Figure 1B) and PBMetaD (Figure 1C) profiles. This shows that partitioning CVs into families does not introduce additional errors, for this system. In fact, on average, PBMetaDPF converges to the reference PT profile approximately three times faster than PBMetaD, as shown by the overlap between the red and green lines in Figure 1B. A three-fold acceleration in convergence is attributed to the fact that the bias potential in PBMetaDPF is constructed by three CVs as opposed to a single CV in PBMetaD.

\section{3-PARTICLE LENNARD-JONES SYSTEM}

To further demonstrate the accelerated convergence offered by the PBMetaDPF, we simulated a 13-particle LJ system $(\sigma=0.39 \mathrm{~nm}, \varepsilon=11 \mathrm{~kJ} / \mathrm{mol})$. A lower $\varepsilon$ value was chosen so that PT simulations could converge in a reasonable amount computational time. In PBMetaDPF, all interatomic distances $(78 \mathrm{CVs})$ were biased and grouped into the same family. Again, we performed 16 independent simulations using the same bias parameters as the three-particle system. We also performed a PT simulation of the system (see details in SI) to provide an independent reference for the free-energy profiles. 

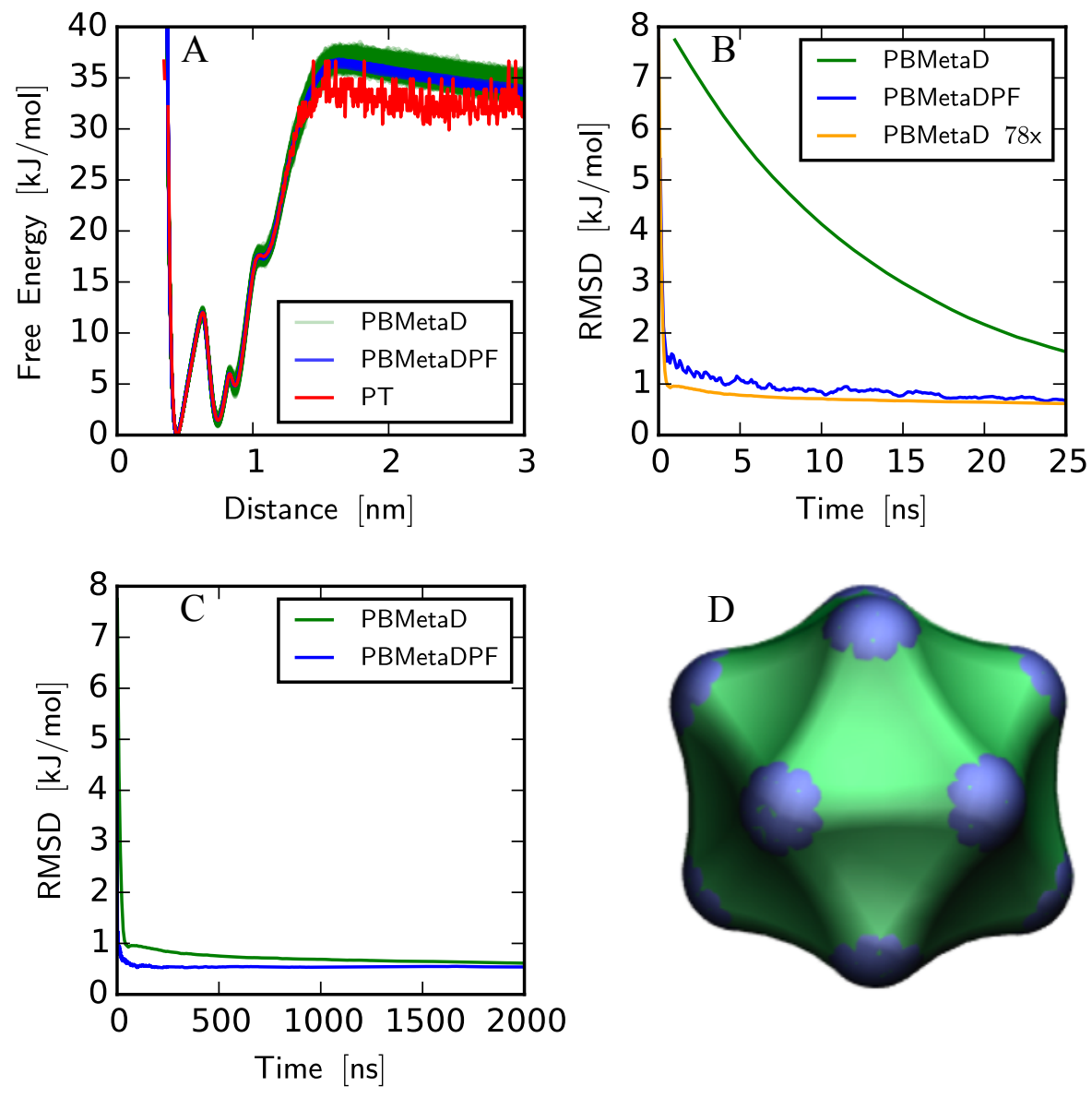

Figure 2. (A) All of the mean-aligned free-energy profiles for PBMetaD after $4 \mu \mathrm{s}$ ( 78 profiles $\mathrm{x}$ 16 trials) and PBMetaDPF after $4 \mu$ s (16 trials) and one profile from parallel tempering (PT). (B) The average RMSD, with respect to the converged PT profile, of PBMetaDPF profiles (blue), PBMetaD profiles (green), and of PBMetaD with a projected convergence rate of 78 times faster (orange) over the course of the simulation. (C) The average RMSD of PBMetaD profiles (green) and PBMetaDPF profiles (blue), all RMSD calculations are relative to the converged PT profile over the course of the simulation. (D) Structure corresponding to the global free-energy minimum. 
PBMetaD (all $78 \mathrm{CVs}$ ) and PBMetadPF converged to the same free-energy profiles (Figure 2A). However, the PBMetaD profiles in Figure 2A exhibit more fluctuations between free-energy profiles than the single PBMetaDPF profile. This highlights the challenge of converging 78 independent free-energy profiles, which necessitates that each atom pair explore the entire CV space. However, we do observe that in the long-time limit (>2 2 s), both PBMetaD and PBMetaDPF give approximately equivalent average RMSD values (Figure 2B). Similar to the LJ3 system, it is shown that partitioning the CVs into a single family allows accelerated convergence of the free-energy profile to (Figure 2C). Most notably, the speed in convergence is again proportional to the number of $\mathrm{CVs}$ in the family, 78 in this case.

In addition to recovering the free-energy with respect to the biased CVs (interatomic distances), the coordinates from the trajectory and metadynamics biases can be utilized to obtain stable structures of the LJ particle system. To find the most stable structure, the frames of the PBMetaD and PBMetaDPF trajectories were clustered (see SI Methods). The clusters were reweighted (described in detail in the SI Methods) using the PBMetaD and PBMetaDPF bias and the method of Torrie and Valleau ${ }^{3}$ to find the most stable structure - an icosahedron (Figure 2D), which had highest probability of all the structures $(\sim 100 \%)$. This result is consistent with previous analyses of the system where the icosahedron structure of $\mathrm{LJ}_{13}$ was shown to be at least $2.85 \varepsilon(=31.35 \mathrm{~kJ} / \mathrm{mol}$ for this system $)$ more stable than other structures. ${ }^{22,23}$

Before this, a MetaD approach that biases $78 \mathrm{CVs}$ for exploring the structural minima of aggregating systems such as the one above would have been intractable. But by partitioning the CVs into a single family, scaling this approach to larger systems is now possible. 


\section{7-PARTICLE LENNARD JONES SYSTEM}

Lastly, to demonstrate the effectiveness of PBMetaDPF to explore and describe systems with multiple, metastable states, we applied it to a 7-particle LJ system constrained to two dimensions. This system is well-studied using a variety of methods and is known to have four stable structural minima and 19 transition states. ${ }^{24-26}$ Here, we apply PBMetaD and PBMetaDPF to explore the potential energy landscape by biasing the 21 interatomic distances, running each simulation for $2 \mu \mathrm{s}$. As in previous treatments of interatomic distances, we partition them into a single PF for PBMetaDPF. For comparison, we also carried out unbiased MD simulations as well as a WTMetaD simulation where the second and third moments of the coordination numbers were biased, following the work of Nava et al. ${ }^{24}$ Both PBMetaD and PBMetaDPF yielded identical free-energy profiles for the interatomic distances. These profiles were identical to the free-energy profiles recovered from reweighting the WTMetaD onto the interatomic distances (Figure 3A). In this case too, PBMetaDPF could converge the free-energy surface for the interatomic distances faster (Figure 3B), and the degree of acceleration is commensurate with the reduction of profiles (21 times faster). Unsurprisingly, the unbiased MD simulation $(\sim 2 \mu \mathrm{s})$ did not generate the correct free-energy surface (Figure SI 2) for this system emphasizing the need for enhanced sampling techniques. 

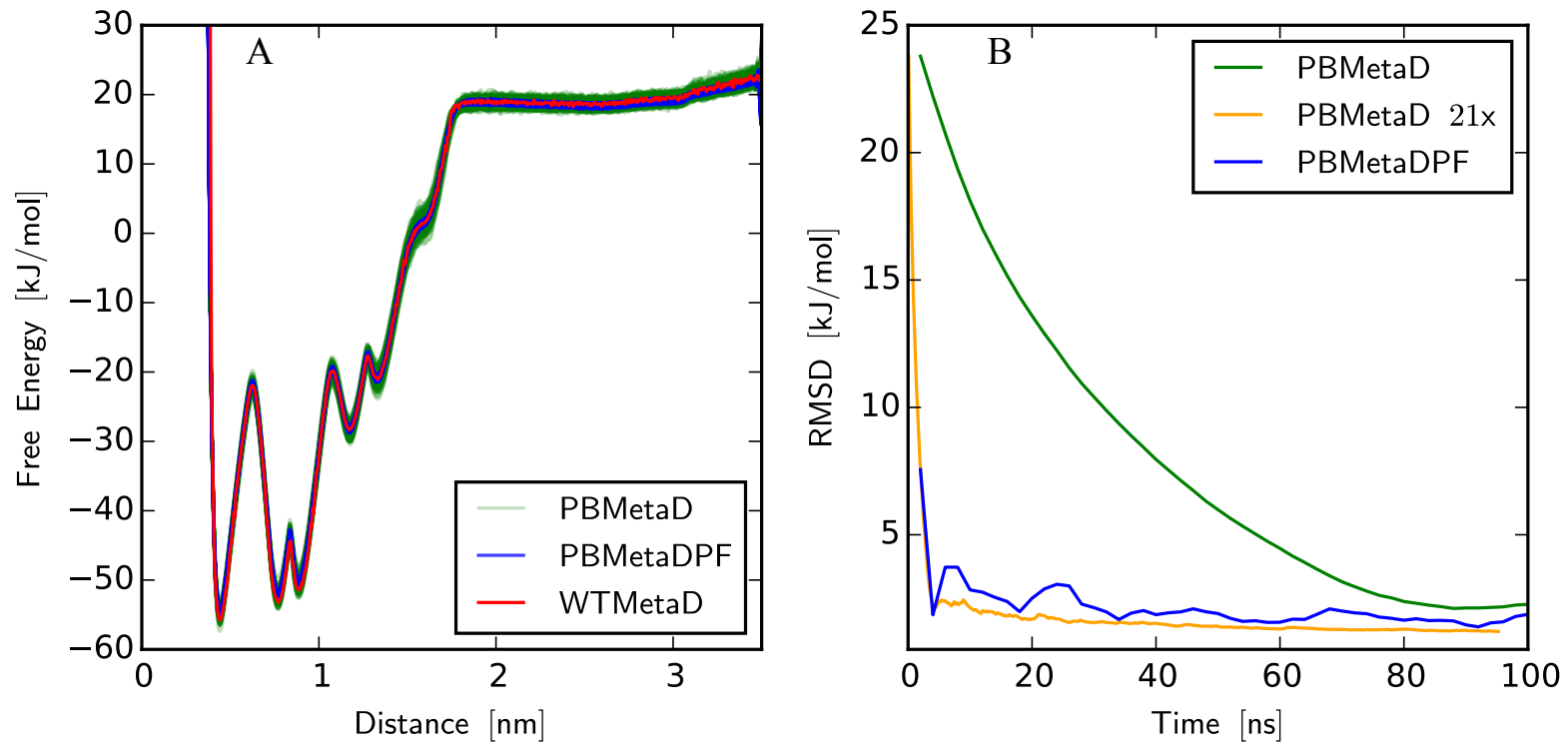

Figure 3. (A) Mean-aligned free-energy profiles of the interatomic distance between LJ particles. In total, the $16 \mathrm{PBMetaDPF}$ profiles, the $336 \mathrm{PBMetaD}$ profiles, and one WTMetaD profile (reweighted) are plotted. (B) The average RMSD of PBMetaDPF profiles (blue), PBMetaD profiles (green), and average RMSD of PBMetaD projected to converge 21 times faster (orange) relative to the converged WTMetaD profile over the course of the simulation. The area of interest was restricted to be $100 \mathrm{~kJ} / \mathrm{mol}$ of the minimum of the reweighted WTMetaD profile.

In previous MetaD studies of this system, the second and third moments of the coordination number were selected and biased, as these CVs were able to differentiate between the four stable structural minima. ${ }^{24,25}$ The limit to two CVs was also more amenable to methods that were unable to bias more than a few CVs at the same time. However, we chose to bias the interatomic distances using the PBMetaD and PBMetaDPF frameworks since it is a far more general approach and the CVs are more interpretable. After the simulations were converged, we reweighted the second and third moments of the coordination number to demonstrate the 
consistency of our results with prior work. ${ }^{24,25}$ As shown in Figure 4A-C, the correct FESs were recovered with reweighting using both PBMetaD and PBMetaDPF. The FESs recovered using the two methods are identical in shape (Figure SI 2) and exhibit identical approach to convergence over the course of the simulation (Figure $4 \mathrm{~B}$ and $\mathrm{C}$ ).
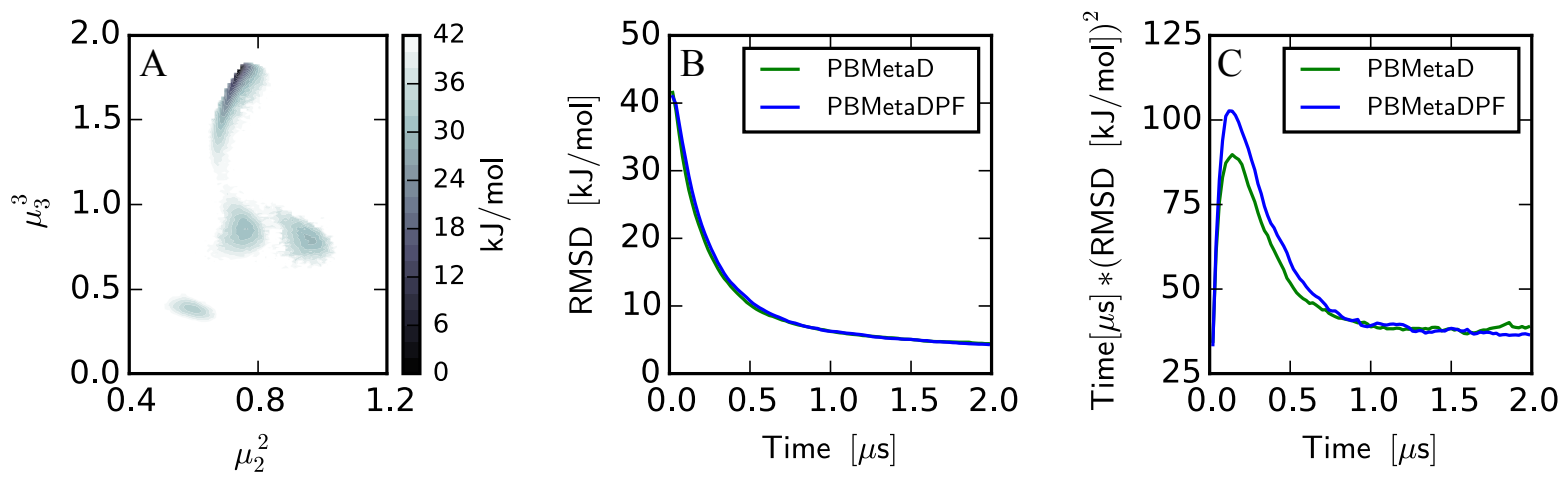

Figure 4. (A) Free-energy surface for the 7-particle LJ system reweighted for the second and third moments of coordination numbers using PBMetaDPF. (B) The average RMSD from 16 PBMetaD and PBMetaDPF simulations (each) reweighted for the second and third moments of coordination numbers with respect to a WTMetaD simulation biasing those same CVs. (C) A demonstration of the absence of systematic error in reweighting both PBMetaD and PBMetaDPF. The area of interest was restricted to be $40 \mathrm{~kJ} / \mathrm{mol}$ of the minimum of the reweighted WTMetaD surface.

Further, we analyzed the trajectories using the clustering and reweighting method described in the SI to find the most stable structural minima. We were able to obtain the minima obtained in previous investigations of the system (Figure 5). Further, the probabilities of the structural minima predicted using PBMetaD and PBMetaDPF were within $0.5 \%$ of each other (Table SI 1). 

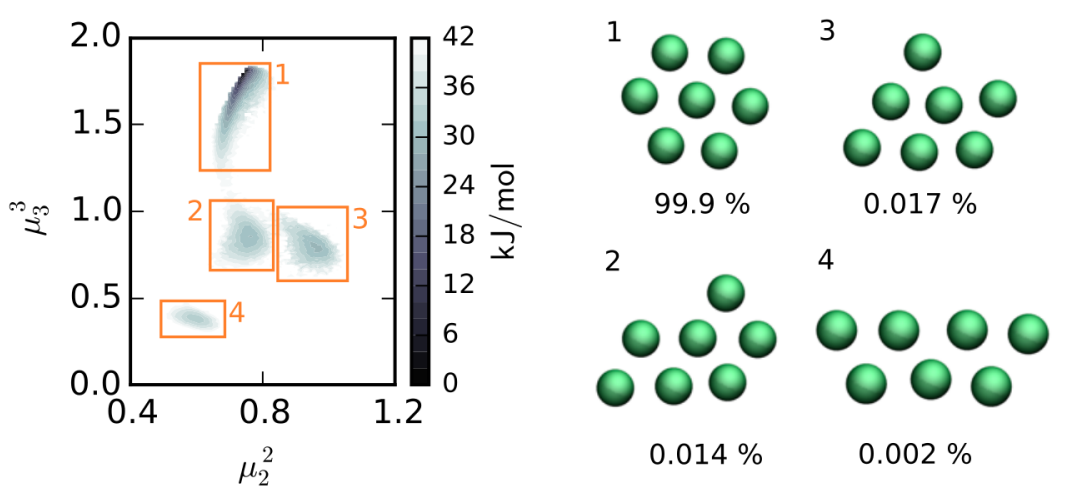

Figure 5. (left) Free-energy surface recovered from PBMetaDPF simulation of the 2D 7-particle LJ system after reweighting for second and third moments of the coordination number. (right) Representative structures for the regions highlighted in orange on the free-energy surface along with the probability of occurrence of each structure in the $2 \mathrm{D}$ phase space plotted on the right.

This study highlights that PBMetaDPF can be used to effectively sample the simple yet challenging structural phase space of the 7-particle LJ system. Furthermore, biasing the interatomic distances is a far more general, and intuitive approach for studying these complex structures. This eliminates the use of unintuitive CVs like moments of coordination numbers. Moreover, unlike PBMetaD, PBMetaDPF offers a more scalable approach by leveraging the excessive number of degenerate CVs to expedite converging an individual FES, rather than converging 21 profiles independently (as also proved in the $\mathrm{LJ}_{13}$ system). Lastly, the free-energy surface with respect to other CVs of interest can always be reweighted and constructed after converging the FES for the distance CVs.

\section{OTHER POTENTIAL APPLICATIONS}

In this study, we have applied PBMetaDPF to simple LJ systems. However, we can predict several other applications for this method where researchers were restricted to using a 
single CV because previous methods were not amenable to multiple, degenerate CVs. In a recent study of ion-channel dynamics, ${ }^{14}$ the authors treated degenerate ion distances as separate CVs in bias-exchange metadynamics and noted that the result was the same. Conceivably, the PBMetaDPF scheme could be used to bias these degenerate variables. A similar use case could be obtaining the potential of mean force (PMF) profiles of ion-pairing systems, using distances between ions as their $\mathrm{CV} .{ }^{27}$ Most ion PMFs are calculated with only a single ion-pair in water which requires an additional correction term to account for differences between simulated (one pair) and experimental ion concentrations. ${ }^{28}$ PBMetaDPF can be used in simulations to bias the distances with multiple ions pairings, treating unique ion-pair distances as PFs, which would allow the recovery of free-energy estimates at realistic concentrations, potentially providing efficient routes to describe ion solvation/desolvation. ${ }^{29}$

Another potential area of application is in aggregation studies, where the free-energy of association of a particle is computed to predict the behavior of aggregates at higher concentrations for experiments. For example, the dimerization free-energy of peptoids in the dilute limit was used to predict how peptoids at higher concentrations aggregate in solution. ${ }^{30}$ Similarly, the dimerization free of two $\mathrm{MgO}$ nanoparticles in vacuum was calculated to understand the crystal growth that occurs in saturated solutions. ${ }^{31}$ We suggest that more crowded environments should be simulated and all the inter-particle distances should be biased to recover better estimates of the free energies of association at realistic, experiment level concentrations. While the concentration levels would typically require biasing too many CVs, partitioning the families of indistinguishable particle interactions should allow for this type of approach to be feasible. Many aggregation studies simulate multiple particles in a box, for example when looking at amyloid peptide aggregation with MD simulations. ${ }^{32,33}$ We propose that using 
PBMetaDPF to bias inter-particle distances, and radius of gyration of all residues exhaustively sample the system and reduce the time required to sample all structural minima.

\section{CONCLUSION}

In this letter, we presented PBMetaDPF, an extension of the PBMetaD approach to expedite convergence for a special sub-class of enhanced sampling problems. The method partition degenerate CVs into families, such that they contribute to the same bias potential, in the spirit of the multiple walker scheme. For 3-, 7-, and 13-particle LJ systems, we demonstrated that partitioning CVs into one family led to accelerating the convergence of free-energy profiles. Further, in the case of the 7-particle LJ system, we show that standard reweighting techniques can be used to calculate the free-energy as a function of other CVs not directly biased and detect stable structural minima. Thus, even after partitioning CVs into families, the method maintains its compatibility with commonly-used reweighting protocols. Finally, and most importantly, grouping the CVs into one family led to an increase in the convergence speed of the free-energy profiles that was proportional to the number of CVs grouped into the family. Lastly, PBMetaDPF has been implemented in the open-source PLUMED ${ }^{34}$ library (developers' version soon to be made available to the public), allowing for this approach to not only be readily accessible, but also be easily applied to variety of systems and problems.

\section{ASSOCIATED CONTENT}

Supporting Information. The supplementary information provided free of charge includes information about the simulation setup, parallel tempering and metadynamics parameters, 
clustering and reweighting analyses, and convergence of the free-energy profiles of the 7-particle Lennard-Jones system.

\title{
AUTHOR INFORMATION
}

\section{Corresponding Author}

*jpfaendt@uw.edu

\section{Author Contributions}

The manuscript was written through contributions of all authors. All authors have given approval to the final version of the manuscript. $\$$ These authors contributed equally. (match statement to author names with a symbol)

\section{Funding Sources}

This work was accomplished with support of AFOSR Grant FA9550-16-1-0051 monitored by Dr. Chiping Li. This work is supported in part by an NSF IGERT grant DGE-1258485. This work was also supported by the MS3 (Materials Synthesis and Simulation Across Scales) Initiative at Pacific Northwest National Laboratory (PNNL), a multi-program national laboratory operated by Battelle for the U.S. Department of Energy. This work was facilitated through the use of computational, storage, and networking infrastructure provided by the Hyak supercomputer system, supported in part by the University of Washington.

\author{
ABBREVIATIONS \\ CV, Collective Variable; PBMetaD, Parallel Bias Metadynamics; PBMetaDPF, Parallel Bias \\ Metadynamics with Partitioned Families; PT, Parallel Tempering; LJ, Lennard-Jones; MetaD,
}


Metadynamics; MD, Molecular Dynamics; RMSD, Root Mean Square Deviation; WTMetaD, Well-Tempered Metadynamics; FES, Free-energy Surface.

\section{REFERENCES}

(1) Laio, A.; Parrinello, M. Escaping Free-Energy Minima. Proc. Natl. Acad. Sci. U. S. A. 2002, $99(20), 12562-12566$.

(2) Barducci, A.; Bussi, G.; Parrinello, M. Well-Tempered Metadynamics: A Smoothly Converging and Tunable Free-Energy Method. Phys. Rev. Lett. 2008, 100 (2), 020603.

(3) Torrie, G. M.; Valleau, J. P. Nonphysical Sampling Distributions in Monte Carlo FreeEnergy Estimation: Umbrella Sampling. J. Comput. Phys. 1977, 23 (2), 187-199.

(4) Voter, A. F. Hyperdynamics: Accelerated Molecular Dynamics of Infrequent Events. Phys. Rev. Lett. 1997, 78 (20), 3908-3911.

(5) Valsson, O.; Parrinello, M. Variational Approach to Enhanced Sampling and Free Energy Calculations. Phys. Rev. Lett. 2014, 113 (9), 1-5.

(6) Comer, J.; Gumbart, J. C.; Hénin, J.; Lelièvre, T.; Pohorille, A.; Chipot, C. The Adaptive Biasing Force Method: Everything You Always Wanted To Know but Were Afraid To Ask. J. Phys. Chem. B 2015, 119 (3), 1129-1151.

(7) Gil-Ley, A.; Bussi, G. Enhanced Conformational Sampling Using Replica Exchange with Collective-Variable Tempering. J. Chem. Theory Comput. 2015, 11 (3), 1077-1085.

(8) Noé, F.; Clementi, C. Kinetic Distance and Kinetic Maps from Molecular Dynamics Simulation. J. Chem. Theory Comput. 2015, 11 (10), 5002-5011. 
(9) Tribello, G. A.; Cuny, J.; Eshet, H.; Parrinello, M. Exploring the Free Energy Surfaces of Clusters Using Reconnaissance Metadynamics. J. Chem. Phys. 2011, 135 (11), 114109.

(10) Tiwary, P.; Berne, B. J. Spectral Gap Optimization of Order Parameters for Sampling Complex Molecular Systems. 2015, 113 (11), 2839-2844.

(11) Raiteri, P.; Laio, A.; Gervasio, F. L.; Micheletti, C.; Parrinello, M. Efficient Reconstruction of Complex Free Energy Landscapes by Multiple Walkers Metadynamics. J. Phys. Chem. B 2006, $110(8), 3533-3539$.

(12) Hošek, P.; Toulcová, D.; Bortolato, A.; Spiwok, V. Altruistic Metadynamics: Multisystem Biased Simulation. J. Phys. Chem. B 2016, 120 (9), 2209-2215.

(13) Šućur, Z.; Spiwok, V. Sampling Enhancement and Free Energy Prediction by the Flying Gaussian Method. J. Chem. Theory Comput. 2016, 12 (9), 4644-4650.

(14) Domene, C.; Barbini, P.; Furini, S. Bias-Exchange Metadynamics Simulations: An Efficient Strategy for the Analysis of Conduction and Selectivity in Ion Channels. J. Chem. Theory Comput. 2015, 11 (4), 1896-1906.

(15) Pfaendtner, J.; Bonomi, M. Efficient Sampling of High-Dimensional Free-Energy Landscapes with Parallel Bias Metadynamics. J. Chem. Theory Comput. 2015, 11 (11), $5062-5067$.

(16) Prakash, A.; Sprenger, K. G.; Pfaendtner, J. Essential Slow Degrees of Freedom in Protein-Surface Simulations: A Metadynamics Investigation. Biochem. Biophys. Res. Commun. 2017, 498 (2), 274-281. 
(17) Heller, G. T.; Aprile, F. A.; Bonomi, M.; Camilloni, C.; De Simone, A.; Vendruscolo, M. Sequence Specificity in the Entropy-Driven Binding of a Small Molecule and a Disordered Peptide. J. Mol. Biol. 2017, 429 (18), 2772-2779.

(18) Bonomi, M.; Camilloni, C.; Vendruscolo, M. Metadynamic Metainference: Enhanced Sampling of the Metainference Ensemble Using Metadynamics. Sci. Rep. 2016, 6 (1), 31232 .

(19) Fu, C. D.; Pfaendtner, J. Lifting the Curse of Dimensionality on Enhanced Sampling of Reaction Networks with Parallel Bias Metadynamics. J. Chem. Theory Comput. 2018, 14, $2516-2525$.

(20) Tiwary, P.; Parrinello, M. A Time-Independent Free Energy Estimator for Metadynamics. J. Phys. Chem. B 2015, 119 (3), 736-742.

(21) Branduardi, D.; Bussi, G.; Parrinello, M. Metadynamics with Adaptive Gaussians. $J$. Chem. Theory Comput. 2012, 8 (7), 2247-2254.

(22) Doye, J. P. K.; Miller, M. A.; Wales, D. J. Evolution of the Potential Energy Surface with Size for Lennard-Jones Clusters. J. Chem. Phys. 1999, 111 (18), 8417-8428.

(23) Pietrucci, F.; Andreoni, W. Graph Theory Meets Ab Initio Molecular Dynamics: Atomic Structures and Transformations at the Nanoscale. Phys. Rev. Lett. 2011, 107 (8), 085504.

(24) Nava, M.; Palazzesi, F.; Perego, C.; Parrinello, M. Dimer Metadynamics. J. Chem. Theory Comput. 2017, 13 (2), 425-430.

(25) Tribello, G. A.; Ceriotti, M.; Parrinello, M. A Self-Learning Algorithm for Biased 
Molecular Dynamics. Proc. Natl. Acad. Sci. U. S. A. 2010, 107 (41), 17509-17514.

(26) Wales, D. J. Discrete Path Sampling. Mol. Phys. 2002, 100 (20), 3285-3305.

(27) Fennell, C. J.; Bizjak, A.; Vlachy, V.; Dill, K. A. Ion Pairing in Molecular Simulations of Aqueous Alkali Halide Solutions. J. Phys. Chem. B 2009, 113 (19), 6782-6791.

(28) General, I. J. A Note on the Standard State's Binding Free Energy. J. Chem. Theory Comput. 2010, 6 (8), 2520-2524.

(29) Laage, D.; Hynes, J. T. On the Residence Time for Water in a Solute Hydration Shell: Application to Aqueous Halide Solutions. J. Phys. Chem. B 2008, 112 (26), 7697-7701.

(30) Ma, X.; Zhang, S.; Jiao, F.; Newcomb, C. J.; Zhang, Y.; Prakash, A.; Liao, Z.; Baer, M. D.; Mundy, C. J.; Pfaendtner, J.; et al. Tuning Crystallization Pathways through Sequence Engineering of Biomimetic Polymers. Nat. Mater. 2017, 16 (7), 767-774.

(31) Spagnoli, D.; Banfield, J. F.; Parker, S. C. Free Energy Change of Aggregation of Nanoparticles. J. Phys. Chem. C 2008, 112 (38), 14731-14736.

(32) Mo, Y.; Lu, Y.; Wei, G.; Derreumaux, P. Structural Diversity of the Soluble Trimers of the Human Amylin(20-29) Peptide Revealed by Molecular Dynamics Simulations. $J$. Chem. Phys. 2009, 130 (12), 125101-212413.

(33) Buchanan, L. E.; Dunkelberger, E. B.; Tran, H. Q.; Cheng, P.-N.; Chiu, C.-C.; Cao, P.; Raleigh, D. P.; de Pablo, J. J.; Nowick, J. S.; Zanni, M. T. Mechanism of IAPP Amyloid Fibril Formation Involves an Intermediate with a Transient -Sheet. Proc. Natl. Acad. Sci. 2013, 110 (48), 19285-19290. 
(34) Tribello, G. A.; Bonomi, M.; Branduardi, D.; Camilloni, C.; Bussi, G. PLUMED 2: New Feathers for an Old Bird. Comput. Phys. Commun. 2014, 185 (2), 604-613.

For Table of Contents Only

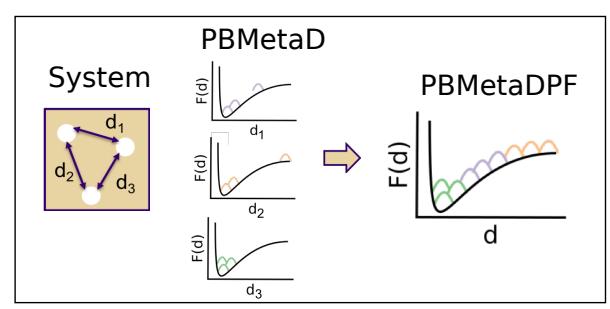

